\title{
Mythologie et « naïveté épique » dans Histoire de Claude Simon
}

\section{Antonin Wiser}

\section{(2) OpenEdition}

1 Journals

Édition électronique

URL : http://journals.openedition.org/ccs/673

DOI : $10.4000 /$ ccs. 673

ISSN : 2558-782X

Éditeur :

Presses universitaires de Rennes, Association des lecteurs de Claude Simon

\section{Édition imprimée}

Date de publication : 31 décembre 2010

Pagination : 49-63

ISBN : 9782354120771

ISSN : $1774-9425$

\section{Référence électronique}

Antonin Wiser, " Mythologie et "naïveté épique » dans Histoire de Claude Simon », Cahiers Claude Simon [En ligne], 6 | 2010, mis en ligne le 21 septembre 2017, consulté le 20 avril 2019. URL : http:// journals.openedition.org/ccs/673; DOI : 10.4000/ccs.673 


\title{
Mythologie et « naïveté épique " dans Histoire de Claude Simon
}

\author{
Antonin WISER \\ Universités de Lausanne et de Paris IV Sorbonne
}

«Pour eux aussi l'Histoire n'était qu'un conte pareil à ceux qu'on a trop entendus."

Joyce, Ulysse.

\section{Préambule}

La mythologie antique hante l'univers romanesque de Claude Simon. Ce constat, que dressera volontiers tout lecteur familier des textes simoniens, vaut tout particulièrement pour le roman qui nous occupera ici. L'écriture d'Histoire semble en effet enregistrer un phénomène que notaient Theodor W. Adorno et Max Horkheimer en 1944, à savoir que "dans le monde rationalisé, la mythologie a envahi le domaine profane $\|^{1}$. On rencontre ainsi dans le roman de Simon un Minotaure tapi au cœur d'une banque, un Sphinx en complet anthracite accordant des prêts hypothécaires, un Hercule

1 Th. W. Adorno, M. Horkheimer, La Dialectique de la raison (1944), trad. française par E. Kaufholz, Paris, Gallimard, 1974, p. 44. 
enfermé dans une case de bande dessinée, ou encore ce Thésée apparaissant «sous l'aspect d'un gringalet gominé et armé d'une mitraillette "(Hist., p.71) ${ }^{2}$.. L'Histoire n'est pas seule à prendre les traits d'une farce, lorsqu'elle se répète : la quasi-citation du $18 \mathrm{bru}$ maire de Marx, qu'on trouve sous la plume du romancier ${ }^{3}$, vaut également pour ces nombreux « retours du refoulé » mythologique.

Mais si la présence du mythe est assez évidente à la lecture d' Histoire, ses fonctions, dans ce roman, le sont beaucoup moins. L'une d'entre elles nous renvoie à la tension qui structure le récit entre un axe horizontal - celui de la journée racontée - et une série de trouées verticales qui nous font plonger dans l'épaisseur de l'histoire, c'està-dire tout à la fois dans le passé personnel du narrateur et dans un passé bien plus lointain. L'insertion des images mythologiques fonctionne alors comme une remise en cause de la progression linéaire et irréversible du temps, pour se faire l'indice d'une certaine répétition historique. Le présent de la journée contée s'emplit alors de ces échos lointains qui viennent brouiller la distinction entre présent et passé et, partant, rendre problématique l'identité comme présence à soi du narrateur. La linéarité narrative s'en trouve également affectée et la cohésion textuelle doit dès lors être prise en charge par des dispositifs autres que la logique pragmatique (logique de l'agir).

\section{Le concept adornien de "naïveté épique "}

J'examinerai la construction du texte de Claude Simon à partir du concept de "naïveté épique » que forgea Adorno dans un texte de 1943. Il s'agira de proposer, par là, un éclairage particulier d'Histoire, mais également d'éprouver en retour la pertinence et la fécondité de ce concept adornien pour l'analyse littéraire.

Ecrit en marge des travaux préparatoires à la rédaction de $L a$ Dialectique de la Raison ${ }^{4}$, "La naïveté épique » traite de la spécificité

${ }^{2}$ Tous les numéros de pages donnés entre parenthèses, précédées de l'abréviation Hist., renvoient à Histoire, Paris, Minuit, 1967.

${ }^{3}$ «Quand elle se répète pour la deuxième fois plus rien qu'une farce Et la combienième maintenant " (Hist., p. 352).

${ }^{4}$ Th. W. Adorno, «La naïveté épique », dans Notes sur la littérature, trad. française par S. Miiller, Paris, Flammarion, 1984, p. 31-36. 
du récit narratif à partir d'une réflexion sur l'épique. Cette notion prend ici une dimension qui déborde le cadre générique de l'épopée proprement dite et se trouve déterminée selon trois aspects que j'exposerai rapidement.

Premier aspect : l'intention épique se comprend à partir de son opposition au mythique. Alors que "le flux amorphe du mythe, c'est l'éternellement semblable ", le mouvement épique désigne un travail de différenciation des objets narrés, dans la mesure où l'épopée - mais ceci est vrai de tout récit - veut parler de « quelque chose qui soit digne d'être raconté [...], qui ne soit pas interchangeable, et qui mérite d'être rapporté en son nom propre $»^{5}$. L'épique s'attache donc au particulier, pour le sauver de sa dissolution dans l'indifférenciation du Même. Pour Adorno et Horkheimer, tel est précisément ce dont parle 1'Odyssée : en luttant contre les puissances archaïques du mythe qui menacent son individuation, Ulysse affirme son identité subjective. L'épopée homérique met ainsi son propre geste en abyme : en racontant la naissance d'un sujet épique (sur le plan diégétique : Ulysse gagne son identité, au terme de son long voyage), la narration construit son propre objet épique, l'histoire déterminée d'un particulier.

Comme geste antimythologique, la narration épique participe du mouvement d'Aufklärung, au sens large qu'Adorno et Horkheimer donnent à ce terme : celui de "pensée en progrès » visant à « libérer les hommes de la peur et [à] les rendre souverains $\|^{6}$. Ceci revient à dire que l'épique, avant d'être une catégorie esthétique, relève de ce que Jean-François Lyotard nomme le « savoir narratif $»^{7}$, et se trouve considéré par Adorno avant tout comme une forme de connaissance.

Second aspect : comme moment dans le procès de l' Aufklärung, le récit épique est toutefois emporté par la dialectique de la raison. Au regard de la ratio, du penser éclairé élevé au niveau des concepts

\section{${ }^{5}$ Ibid., p. 31.}

${ }^{6}$ La Dialectique de la raison, op. cit., p. 21.

${ }^{7}$ J.-F. Lyotard, La condition postmoderne, Paris, Minuit, 1979, p. 36. On peut noter au passage que la critique de la téléologie rationaliste proposée par Adorno et Horkheimer dans la thèse centrale de la Dialectique de la raison annonce la possibilité de la réévaluation du «savoir narratif» que présente la Condition postmoderne. 
universels, l'attachement épique envers le particulier apparaît cette fois comme un "élément de bêtise ${ }^{8}$, un moment d'aveuglement. Envisagé du point de vue téléologique de la "pensée en progrès », le savoir narratif présente tous les traits d'un degré de conscience inférieur au savoir théorique : tel est le sens de sa naïveté.

Pourtant, derrière l'attachement apparemment borné du savoir narratifau particulier se cache en réalité l'aspiration qui fonde l'utopie de la connaissance, et que la Théorie esthétique formule en ces termes:

L'art corrige la connaissance conceptuelle parce que, séparé, il accomplit ce que celle-ci attend en vain de la relation abstraite sujet-objet : le fait que le travail subjectif fasse apparaître quelque chose d'objectif ${ }^{9}$.

Il semble donc que cette aspiration à rencontrer l'objectif pardelà la clôture monadologique du Sujet soit conservée précisément dans ce qui fait la naïveté de l'épique. Telle serait en effet la portée du geste qui s'efforce de « fixer les choses du passé fidèlement, sans les déformer, $[\ldots]$ et par là de rompre le charme qu'exerce le passé, le mythe au sens propre $»^{10}$. On lit ici le désir d'un langage qui chercherait « à se guérir du négatif de son intentionnalité, de la manipulation conceptuelle des objets et à faire apparaître le réel dans toute sa pureté, préservé de la domination de l'ordre $»^{11}$.

Troisième aspect : ce mouvement, en ce qu'il a de fondamentalement anti-discursif, rencontre cette fois la résistance de son propre médium, le langage. L'épique se voit confiné - par le désir sous-jacent qui l'anime - à sa propre impossibilité, c'est-à-dire à la " folie $»^{12}$ même, au regard de la raison théorique. L'attachement au particulier en ce qui le définit comme non-identique (pour reprendre l'une des catégories de la Dialectique négative), conduit le langage à vouloir 《s'effacer lui-même dans le nom et l'image $»^{13}$. L'impulsion mimé-

\footnotetext{
${ }^{8}$ « La naïveté épique », op. cit., p. 32.

${ }^{9}$ Th. W. Adorno, Théorie esthétique (1970), trad. française par M. Jimenez, Paris, éd. Klincksieck, p. 164.

${ }^{10}$ " La naïveté épique », op. cit., p. 33.

${ }^{11}$ Ibid., p. 34.

${ }^{12}$ Idem, p. 34.

${ }^{13}$ Idem.
} 
tique qui voudrait accorder le primat à l'objet se heurte à l'irréductible dimension discursive et doit alors trouver refuge ailleurs : dans la suspension de la rigidité logique du récit. C'est là ce qu'Adorno désigne comme « l'enchaînement épique » :

[L]a conduite de la pensée finit par se relâcher, devient la grâce qui dans le langage prime le droit de juger [...]. La fuite des idées, la forme du discours sacrifié, c'est la fuite du langage hors de sa prison ${ }^{14}$.

Dans l'affaiblissement de la cohésion syntaxique, l'autonomisation des métaphores, la prépondérance de la description ou encore le renversement du rapport de l'image et de l'action, le récit épique soustrait son objet à sa relève logique par la signification articulée depuis des catégories subjectives. Ainsi peut se maintenir le particulier en tant que particulier, c'est-à-dire en sa non-identité avec le Sujet.

\section{Télémaque et Ulysse en territoire simonien}

Je me risque donc à proposer, dans Histoire, un repérage des trois aspects dégagés du texte d'Adorno.

Le premier, qui concerne la confrontation entre l'effort épique de constitution du particulier et les forces mythiques de sa dissolution, me semble trouver son expression précisément au travers d'une série de scènes qu'on peut rapprocher de séquences-clés de 1'Odyssée. Je propose de lire dans l'intertexte homérique autre chose qu'un simple hommage à Joyce ${ }^{15}$. On peut considérer le moi du narrateur comme ce particulier auquel s'attache le récit. A l'instar du Télémaque homérique ${ }^{16}$, l'identité de ce moi est affectée par l'absence du père. Bien qu'on considère plus volontiers L'Acacia (1989) comme le texte consacré à la quête du père, le manque initial de celui-ci dans Histoire provoque également des effets dont le principal est certainement le déclenchement de la question de l'origine. L'une des toutes

\footnotetext{
${ }^{14}$ Ibid., p. 35.

${ }^{15}$ C'est en effet la lecture que propose Jean Starobinski dans « La journée dans

"Histoire» », dans J. Starobinski et alii, Sur Claude Simon, Paris, éd. Minuit, 1987, p. 12 .

${ }^{16}$ Homère, L'Odyssée, chant I, v. 215-216, traduction de Philippe Jaccotet, Paris, éd. La Découverte, 2004,
} 
premières occurrences de cette question apparaît en effet dans un passage qui évoque la paternité, au détour d'une remarque portant sur le sexe d'un ancien prétendant de la mère, évoqué en des termes pour le moins caricaturaux :

[...] les attributs aujourd'hui pendouillants et ridés de sa défunte virilité [...] les inglorieux débris d'où le hasard aidant j'aurais pu sortir éjecté arraché d'un tressautement de ce corps flasque (Hist., p. 52)

La contingence de son événement, qui apparaît au narrateur dans cette rencontre avec le vieux soupirant, vient alors comme remarquer la place manquante du géniteur et initie un travail archéologique qui va traverser le roman tout entier.

De son père qui, comme Ulysse, fit de si longs et lointains voyages, le narrateur n'a plus pour seules traces que des cartes postales adressées à sa mère et portant souvent pour unique message un nom, une date et un lieu. Que ces « fragments, écailles arrachées à la surface de la vaste terre" (Hist., p. 19) soient insuffisants à produire quelque assurance ontologique que ce soit, ne le sait-on pas depuis Derrida?

c'est le postal, le Principe Postal comme relais différentiel, qui régulièrement empêche, retarde, endépèche le dépôt de la thèse, interdit le repos et sans cesse fait courir, dépose ou déporte le mouvement de la spéculation $^{17}$.

La mort du père n'arrêtera pas sa différance ; le lieu de sa sépulture, indiqué lui-même au moyen d'une carte postale, reste indéterminé, une tombe parmi tant d'autres dans un cimetière de soldats tués au combat:

et pour lui même pas de nom tombe anonyme cette carte que quelqu'un avait envoyé LUZY (Meuze) Cimetière hémicirculaire élevé par les Allemands pendant l'occupation, avec écrit au-dessous de l'écriture penchée épineuse orgueilleuse " pour nos morts et les leurs». (Hist., p. 384-385)

${ }^{17}$ J. Derrida, La carte postale. De Socrate à Freudet au-delà, Paris, éd. Aubier-Flammarion, 1980, p. 61. Poséidon n'est au fond pas autre chose pour 1'Odyssée qu'une personnification - ou une déification - de ce principe de différance du retour. 
C'est donc là, dans cette absence irrémédiable du géniteur, que je lis l'origine - qui n'en est dès lors plus vraiment une - d'une quête épique de l'origine, dont on sait qu'elle conduira le lecteur au dernier mot de l'histoire : "moi ?... " \{Hist., p. 402). Que ce " résultat" soit précisément problématique, c'est là ce sur quoi il faudra s'interroger plus loin, mais cette incertitude ne fait pas obstacle à l'intention épique du récit, qui vise à arracher au moi particulier une identité en interrogeant sa genèse.

Dans L'Odyssée, l'identité de l'individu est menacée par les puissances mythiques qui invitent Ulysse et ses compagnons à goûter au plaisir d'une régression vers le passé préindividuel. L'appel est émis plus particulièrement depuis trois lieux : l'île des Lotophages, celle des Sirènes et celle de Circé.

Adorno rattache l'île des Lotophages à « la tentation de régresser à un stade où l'homme cueillait les fruits de la terre et de la mer $»^{18}$. En ce pays de Cocagne, les hommes oublient tout désir de retour ${ }^{19}$, c'est-à-dire qu'ils se détachent de la temporalité vectorielle de l'épopée. Cette suspension temporelle au sein d'un paradis terrestre nous renvoie aux dernières évocations de la mère, dans la douzième section d'Histoire: "Bourrée de morphine ", "somnol[ant] presque tout le temps " dans une chambre qu'elle a fait remplir de fleurs, les cartes postales étalées sur son lit, elle semble «parvenue (ou revenue) à une éternelle félicité parmi les palmiers aux troncs penchés " \{Hist., p. 400) dans un rêve où « le temps [est] hors d'atteinte" :

tout était arrêté maintenant présent immobilisé tout là dans un même moment à jamais les images les instants les voix les fragments du temps du monde multiple fastueux éparpillés sur un lit de mourante. (Hist., p. 385)

Comme le lotus, la morphine promet ici un temps retrouvé hors du temps. "Félicité Island ", tel est le nom que prend, dans Histoire, l'île des Lotophages. Mais si ce nom et ce lieu désignent pour la mère le passé extatique du séjour exotique aux « luxuriants décors de luxuriantes forêts " \{Hist., p.388), c'est également le point de fuite de la régression ontogénétique du narrateur, l'extrême pointe de son

\footnotetext{
${ }^{18}$ La Dialectique de la raison, op. cit., p. 76.

${ }^{19}$ Chant IX, v. 97.
} 
non-lieu, dont il ne peut s'approcher qu'avec incertitude (« ce sein qui déjà peut-être me portait », Hist., p. 401) et par le biais, artificiel, d'une fiction rétrospective (" pouvant croyant la voir », Hist., p. 388) ayant pour seul espace celui que reconstruit l'écriture.

Dans ce regard tourné vers l'origine biologique du moi, on retrouve un écho de la fascination pour le chant des Sirènes. "La séduction qu'elles exercent représente la tentation de se perdre dans le passé $\|^{20}$, commentaient Adorno et Horkheimer. Cette tentation, le narrateur la rencontre sur la route qui le ramène chez lui, à la nuit tombante. Les hésitations de la lumière déclinante lui donnent en effet l'impression d'avoir «non pas avancé progressé mais reculé dans le temps " (Hist., p. 321) avant de subir à nouveau l'épreuve de l'expulsion primordiale :

comme si j'étais tiré à hue et à dia dans le temps plongé brusquement dans l'obscurité m'enfonçant dans les ténèbres puis extirpé repoussé en sens inverse retrouvant la lumière (Hist., p. 323)

Voir le jour à nouveau, comme pour la première fois, à la sortie de cette nuit "semblable à une sorte de matrice " (Hist., p.348), après s'y être abandonné pour renaître à l'aube, en une enfance immaculée, tel est le fantasme régressif qui s'exprime ici :

pensant que je pourrais continuer ainsi avançant toujours m'enfonçant dans ses entrailles tièdes sans rien voir d'autre que les ténèbres rassurantes Tard dans la nuit il $y$ aurait de temps en temps un village endormi ou une ville illuminée [...] puis disparaissant et de nouveau rien que le noir originel et demain matin il $y$ aurait des montagnes l'air pur de la neige un lac avec des voiles doubles des mouettes entrecroisées tout serait de ce bleu à la fois léger et profond les pics les glaciers se reflétant je me rappelle ce bateau à aubes [...] il $y$ aurait des enfants (Hist., p. 324)

Mais le désir de la régression ontogénétique gagne dans le roman une dimension supplémentaire avec le redoublement de la scène d'origine, la reproduction de l'acte sexuel. A l'instant où il approche au plus près de l'analogon de son événement, le moi narrateur est 
renvoyé par-delà sa vie à un passé phylogénétique qui se perd dans la nuit des temps, jusqu'avant l'histoire:

ma pine enfoncée en elle moi étendu rigide mort pouvant me voir sous la forme de ce guerrier ou chasseur ithyphallique de la préhistoire réduit à quelques traits charbonneux comme si j'étais fait de barres raidi tout mon corps aux membres durcis noirs mes os parallèles qu'on retrouverait des siècles plus tard dessinés sur les parois des cavernes (Hist.,

p. 125-126)

En ce guerrier archaïque, tout entier tendu par la pulsion sexuelle, la différence de l'homme et de l'animal s'estompe. Ce qui se joue chez Simon, dans l'érotisme - qu'on se rappelle par exemple la scène d'amour entre Georges et Corinne dans La Route des Flandres ${ }^{21}$ c'est un devenir-animal au sens deleuzien. On peut lire en effet dans Mille plateaux que " la sexualité passe par [...] le devenir-animal de l'humain $»^{22}$. C'est-à-dire, pour Deleuze, par la dissolution de l'identité dans le multiple ${ }^{23}$ : la sexualité déterritorialise le sujet humain. Ce que Deleuze évoquait ainsi au titre de "souvenir d'un sorcier" nous ramène sur les rivages de la magicienne Circé. Il y a là en effet matière à un rapprochement avec le constat suivant d'Adorno : « de tout temps, la forme animale que prennent les hommes séduits a été mise en rapport avec ce retour aux instincts élémentaires et Circé est devenue le prototype de la courtisane $»^{24}$. La scène érotique évoquée par le narrateur dans l' avant-dernière section du roman \{Hist., p. 341-342) donne à voir une telle métamorphose, catalysée par l'arrière-fond intertextuel de L'Ane d'or d'Apulée. On rencontre ainsi " une créature hybride [...] moitié étalon moitié homme », avançant " à la façon d'un caniche » et pourvu d'un "long membre de chien ou de singe ». Le glissement continu de cette scène fantasmée ( imaginant») entre Corinne et le jockey Iglesia vers celle de la défloration remémorée (remembrée ?) par le narrateur (« quand je me

${ }^{21}$ La Route des Flandres, dans Claude Simon, Euvres, Paris, Gallimard « Bibliothèque de la Pléiade », 2006, p. 372-373.

${ }^{22}$ G. Deleuze, F. Guattari, Mille plateaux, Paris, Minuit, 1980, p. 341.

${ }^{23}$ « Dans un devenir-animal, on a toujours affaire à une meute, à une bande, à une population, à un peuplement, brefà une multiplicité », ibid., p. 292.

${ }^{24}$ La Dialectique de la raison, op. cit., p. 82. 
retirai d'elle ») évoque une régression qui entraîne le moi en deçà du principe d'individuation.

Mais d'une façon peut-être plus radicale encore, le devenir-animal au cœur de l'acte sexuel vient éveiller, chez le narrateur qui en est issu, une doute sur sa propre humanité. Un doute que renforce la morphologie animalière des divers personnages (on pense tout particulièrement aux femmes-oiseaux et aux hommes-coléoptères) et qui culmine dans l'interrogation finale de l'excipit d'Histoire: "sorte de têtard gélatineux lové sur lui-même avec ses deux énormes yeux sa tête de ver à soie sa bouche sans dents son front cartilagineux d'insecte, moi ?...» \{Hist., p. 401).

\section{L'épopée défaillante et la crise du Sujet}

A la différence de ce qui advient dans le récit homérique, l'issue de l'épreuve des séductions mythiques est ici très incertaine. Le moi qui surnage à la surface de l'axe horizontal - le récit de la journée est rendu problématique par les nombreuses trouées anamnésiques qui le replongent dans les profondeurs de l'histoire individuelle et collective. En ce sens, il n'est pas certain que d'Histoire naisse un véritable sujet épique, au sens d'un particulier tout à fait différencié et individué. Mais dans cette difficulté même, le roman enregistre l'expérience de la crise du sujet moderne. Et c'est le traitement strictement narratif et esthétique de cette expérience qui relève de la naïveté épique proprement dite.

La crise des Grands Récits - dont l'épopée fait assurément partie - ébranle la téléologie du Sujet et avec lui le concept d'expérience qui, depuis la Phénoménologie de l'Esprit ${ }^{25}$, lui est étroitement lié. C'est la possibilité même d'une intériorisation/assimilation - l'Errin-

${ }^{25}$ Voir Hegel, Phénoménologie de l'Esprit, « Préface » : « Et l'on appelle expérience justement ce mouvement où l'immédiat, le non-expérimenté, i.e. l'abstrait, que ce soit l'être sensible ou simplement pensé, s'aliène, et ensuite fait retour sur soi à partir de cette aliénation, et du coup se présente maintenant seulement dans son effectivité et vérité, tout comme il est propriété de la conscience " (trad. française par G. Jarczyk et P-J. Labarrière, Paris, Gallimard, 1993, p. 97). L'expérience de la conscience est la relève (Aufhehung) par quoi le savoir de la vérité se hisse au niveau de l'Esprit absolu. 
nerung hégélienne - de l'expérimenté en une conscience subjective identique à elle-même que l'extrême modernité affecte profondément. Dans un texte paru en 1954 et portant sur la situation du narrateur dans le roman contemporain, Adorno résume la situation nouvelle en ces termes : "l'identité de l'expérience est brisée, la vie continue et articulée qui seule autorise l'attitude du narrateur », en évoquant aussitôt la situation que décrivait déjà Benjamin à la sortie de la Grande Guerre : "il suffit de se représenter l'impossibilité pour quelqu'un qui a participé à la guerre de la raconter comme on pouvait autrefois raconter ses aventures $»^{26}$. Cette impossibilité est thématisée, on le sait ${ }^{27}$, au cœur même d'Histoire par le personnage de l'oncle Charles :

ce qui fait qu'il n'est pas plus possible de raconter ce genre de choses qu'il n'est possible de les éprouver de nouveau après coup, et pourtant tu ne disposes que de mots, alors tout ce que tu peux essayer de faire...

(Hist., p. 152)

Dans ce "pourtant», on peut lire l'insistance obstinée et obtuse du langage devant ce qui, manifestement, refuse de s'y laisser enfermer. Cette insistance sur l'événement particulier qui résiste à sa traduction en catégories discursives, ce n'est pas autre chose que ce qu'Adorno considérait comme la naïveté de l'épique. Et que ce " pourtant» donne lieu à une œuvre romanesque, qui enregistre en une forme esthétique la crise de l'expérience, c'est le redoublement de cette obstination naïve. L'impossible accès au récit de l'histoire celle de l'origine ou de l'expérience - continue à s'énoncer comme un savoir narratif.

\section{L'émergence d'un deuxième langage}

Ceci nous conduit à considérer le troisième aspect de la dimension épique, que je ne pourrai aborder ici que brièvement. Il

${ }^{26} \mathrm{Th}$. W. Adorno, « La situation du narrateur dans le roman contemporain », dans Notes sur la littérature, op. cit., p. 38. Voir également W. Benjamin, « Expérience et pauvreté », dans Euvres II, trad. française par P. Rusch, Paris, Gallimard, 2000, p. 364-372.

${ }^{27}$ Voir notamment L. Dallenbach, Claude Simon, Paris, Seuil, 1988, p. 30. 
met en jeu en effet l'ensemble du dispositif scriptural de Claude Simon en tant qu'effort du langage pour parvenir à briser le cercle magique d'une subjectivité qui le coupe de l'objet qu'il cherche à dire. Parmi les nombreux aspects de l'écriture simonienne dont on peut penser qu'ils contribuent à organiser cette " fuite du langage hors de sa prison " dont parlait Adorno, je ne retiendrai que les deux suivants.

Tout d'abord, le lecteur d'Histoire n'aura pas manqué de relever les nombreuses occurrences de remotivations sémantiques qui trahissent ce qu'on pourrait interpréter comme une nostalgie de la langue d'avant la chute de Babel. Lire dans la matérialité du mot veuf l'inscription même de la coupure et du deuil ${ }^{28}$, c'est tout à la fois fantasmer une langue adamique et dans le même geste puisque la remotivation fait l'objet d'une explicitation - constater son inexistence immédiate. Le désir de l'enfant qui cherchait «la réponse à quelque secret capital» (Hist., p. 106) dans les pages des dictionnaires est en définitive déçu. La « communauté magique [du langage humain] avec les choses $»^{29}$ n'est plus, pour autant qu'elle ait jamais eu lieu. L'expérience des mots est celle de leur distance irréductible avec ce qu'ils prétendent dire. Ainsi, pour le mot « sang »:

entre le lire dans les livres ou le voir artistiquement représenté dans les musées et le toucher et recevoir les éclaboussures c'est la même différence qui existe entre voir écrit le mot obus et se retrouver d'un instant à l'autre couché cramponné à la terre et la terre elle-même à la place du ciel et l'air lui-même qui dégringole autour de toi (Hist., p. 152)

Si la naïveté épique conserve malgré tout quelque chose de l'utopie d'un langage qui pourrait franchir la distance entre l'événement et l'énoncé, un langage qui "voudrait s'effacer lui-même dans le nom $»^{30}$, c'est ailleurs qu'elle trouve refuge : dans la constellation

${ }^{28}$ Cf. Hist., p. 82.

${ }^{29} \mathrm{~W}$. Benjamin, "Sur le langage », dans Euvres I, trad. française par M. de Gandillac et R. Rochlitz, Paris, Gallimard, 2000, p. 152.

30 « La naïveté épique », op. cit., p. 34. 
- en un sens benjamino-adornien - qui gravite autour d'un centre absent. Constellation de mots lorsque un nom vient à manquer (ainsi cette périphrase du narrateur d'Histoire, tandis que lui fait défaut le nom des oiseaux perchés dans le grand acacia, " ceux [qui] comment s'appellent-ils avec leurs joues blanches et cette petite calotte bleu-noir sur la tête poussant ce pihihi pihihi discordant ", Hist., p. 230), ou constellation d'images, comme les nombreuses descriptions de cette ville-cadavre pourrissante de Barcelone, qui font signe indirectement vers l'indicible échec de la révolution espagnole. Dans les deux situations, il ne serait pas exagéré de voir " l'exactitude de la parole descriptive [qui] cherche à compenser la non-vérité de tout discours $»^{31}$

Mais c'est surtout dans l'enchaînement textuel selon des principes de contiguïté phonique, iconique ou sémantique que se manifeste cet "effondrement logique du langage épique ", comme autonomisation des images par rapport au récit de l'action. Ce phénomène ne désigne pas la destruction du langage, mais bien plutôt, ainsi qu'Adorno l'écrira quelques années plus tard, la constitution d'un « deuxième langage, souvent à partir du substrat du premier, un langage des choses, effondré, fait d'associations $\|^{32}$.

Je ne prendrai qu'un seul exemple de cette autonomisation d'images qui en viennent à tisser la trame du texte : à la page 366 d'Histoire, l'écriture nous conduit d'un lieu diégétique à un autre, à partir d'évocations de Barcelone. Le train que prend Hélène, se séparant du narrateur, est le BARCELONE-EXPRESS, un nom qui fait surgir une carte postale envoyée par l'amie espagnole de la mère et représentant une vista GENERAL de la ville catalane. Cette "vue» appelle à son tour le souvenir de la guerre d'Espagne et des conflits dont Barcelone fut le théâtre, "pareille à ces arènes à l'intérieur desquelles se déroule un invisible spectacle ». Une image qui évoque alors celle de la mère assistant aux corridas dans sa jeunesse et que le narrateur reconstruit à l'aide de son imagination et de l'« une de ces vieilles photos " jaunies qu'il retrouve avec les cartes dans un tiroir. Cette pratique de la dérive textuelle, on sait que Simon l'a lui-même commentée dans le Discours de Stockholm, en parlant d'une causalité

\footnotetext{
31 Idem.

32 « La situation du narrateur ... », op. cit., p. 43.
} 
« intérieure » au fait littéraire qui prend le pas dans son écriture sur la «causalité d'ordre psycho-social $»^{33}$.

Une logique grammatologique (qui est tout autre chose qu'une irrationalité) l'emporte sur la psycho-logique ou la pragmato-logique, dont les contraintes articulatoires de la narration sont affaiblies. Avec l'émergence de ce " deuxième langage » empreint de naïveté épique, c'est donc tout l'ordre logocentrique qui se trouve contesté et avec lui, on le sait, la préséance accordée au présent. Depuis le lieu graphique de cette contestation remontent alors les spectres de la mythologie, l'antique ou la personnelle, qui viennent hanter la journée d'Histoire et substituer au Sujet maître de soi un moi problématique.

Ce moi problématique et grammatologique - au sens où il n'existe que dans l'écriture qui, comme Pénélope, le tisse et aussitôt le défait -, ce moi qui résiste aux schèmes de l'identité subjective ressemble beaucoup à cet « individuel non-identique » dont Adorno fait le telos de la connaissance. S'il n'est pas le sujet homérique, qui se domine lui-même pour mieux dominer le monde, c'est peut-être précisément parce qu'il entend réparer quelque peu l'injustice que ce sujet épique fait subir à ce qui est différent. C'est la mythologie moderne du sujet rationnel - héritier du rusé Ulysse - que la naïveté épique d'Histoire vient déranger. Ce faisant, cette dernière témoigne de quelque sympathie pour les mythes, victimes de la Raison éclairée, mais sans pour autant leur rendre leur ancienne puissance. On peut voir là un des motifs du recours de Simon au matériau mytho-

${ }^{33}$ C. Simon, Discours de Stockholm, dans Euvres, op. cit., p. 896. Cette remarque devrait suffire à nous garder de projeter trop rapidement sur la dérive ici commentée la représentation, psychologisante s'il en est, des successions de pensées d'un narrateur cherchant le sommeil. Affirmer par ailleurs que «l'on écrit (ou ne décrit) jamais quelque chose qui s'est passé avant le travail d'écrire, mais bien ce qui se produit (et cela dans tous les sens du terme) au cours de ce travail " (ibid., p. 898), c'est bien dire également que les romans de Simon ne sont pas les comptes rendus ou les transcriptions d'exercices de phénoménologie de la conscience (par exemple, l'écriture d'une expérience des états de conscience entre la veille et le sommeil). C'est bien plutôt l'inverse : un dispositif scriptural, qui se laisse guider dans ses associations et enchaînements par la matérialité du langage, devient ensuite un interpretans possible pour des phénomènes de conscience qui, partant, relèvent d'une grammatologie, avant toute psychologie. 
logique, mais aussi y trouver un élément d'élucidation du traitement des mythes dans ses romans, qui s'y voient bien souvent fragmentés, décalés et ironisés.

Le moment de naïveté épique chez Claude Simon opère un geste d'autoréflexion de la logique scripturale et participe d'une critique du renversement de la raison moderne en mythe. Ce moment désigne ainsi, dans Histoire, le contenu de vérité, si tant est que, pour reprendre une formule d'Adorno :

[S]eules sont réussies [...] les œuvres qui sauvent quelque chose de l'amorphe auquel elles font inévitablement violence ${ }^{34}$.

34 Théorie esthétique, op. cit., p. 80. 